Research Note

\title{
Comparison of Different Vegetative Indices for Calibrating Proximal Canopy Sensors to Grapevine Pruning Weight
}

\author{
James A. Taylor ${ }^{1 *}$ and Terence R. Bates ${ }^{2}$
}

\begin{abstract}
Canopy sensing in viticulture is widely associated with the term NDVI (normalized difference vegetation index). However, there are many other vegetative indices (VIs) that can be calculated from information captured with visible/near-infrared (NIR) sensors. A proximal canopy sensor was used to survey 27 vineyards in the Lake Erie Concord belt and stratified to collect pruning weights (PW) at a density of $\sim 25$ samples per vineyard. Seven VIs were derived from the sensor data and the first principal component (PC1) extracted from a principal components analysis of the seven VIs. The VIs and PC1 were regressed against the local PW measurements and ranked in terms of their goodness-of-fit. Over the 27 vineyards, there was no single VI that outperformed the others, although VIs that used the red-edge band had a slight advantage over VIs using the red band. It is therefore recommended to use the normalized difference red edge index (NDRE) in place of the NDVI when predicting PW from terrestrial-based proximal canopy surveys. The PC1 derived from the decomposition of all seven VIs did appear to convey some benefit to PW prediction compared with a single VI approach, particularly with just NDVI. More research into the potential for multivariate approaches is recommended.
\end{abstract}

Key words: CropCircle, normalized difference red edge index (NDRE), normalized difference vegetation index (NDVI), precision viticulture

Multispectral optical sensors are widely available for use in viticulture (Matese et al. 2015). These sensors record the reflectance of light from the canopy at certain wavelength intervals (bands), including visible (blue, green, and red; B, $\mathrm{G}$, and R), red-edge (RE), and the near-infrared (NIR) bands. Sensors typically measure two to four bands, but the width and the center wavelength of bands does differ slightly between sensors. Normally, information from multiple bands is compressed into a single-value vegetation index (VI) to simplify the output. The VIs derived from a sensor will depend

${ }^{1}$ UMR ITAP, University of Montpellier, INRAE, Institut Agro, Montpellier, France; and ${ }^{2}$ Cornell Lake Erie Research and Extension Laboratory, School of Integrative Plant Science, Cornell University, 6592 West Main St, Portland, NY 14769

*Corresponding author (james.taylor@inrae.fr; tel: +33 6176721 55)

Acknowledgments: This work was possible because of the financial support provided by the National Grape Research Alliance (NGRA). The project team would like to express our gratitude to NGRA and the various viticulture organizations that support NGRA. Additional financial support was also provided by the New York Grape and Wine Foundation and the USDA-NIFA Specialty Crop Research Initiative Award No. 2015-51181-24393. The authors would also like to thank all the growers and their employees that were involved and the technical and extension staff at the Cornell Lake Erie Research and Extension Laboratory.

Manuscript submitted July 2020, revised Feb 2021, accepted March 2021

This is an open access article distributed under the CC BY license (https:// creativecommons.org/licenses/by/4.0/).

By downloading and/or receiving this article, you agree to the Disclaimer of Warranties and Liability. The full statement of the Disclaimers is available at http://www.ajevonline.org/content/proprietary-rights-notice-ajev-online. If you do not agree to the Disclaimers, do not download and/or accept this article. doi: 10.5344/ajev.2021.20042 on the bands measured, but most VIs make use of differences in reflectance at certain wavelengths to differentiate between healthy, vigorous plants and unhealthy, stressed plants.

Previous studies have shown the link between VI responses and vine canopy size and/or vigor (Dobrowski et al. 2003, Johnson 2003, Hall et al. 2008, Drissi et al. 2009, Stamatiadis et al. 2010, Taylor et al. 2013). In these studies, the most common VI used has been the normalized difference vegetation index (NDVI) (Rouse et al. 1974). The NDVI was one of the first VIs reported in the agricultural domain when imagery from satellite-based (Landsat series) optical sensors first became available. The NDVI is a normalized combination of the red and NIR reflectance that generates a value between 0 and 1 (details in Table 1).

Over the past $45+$ years, NDVI has been shown to be useful in a wide range of vegetative studies. Optical sensors that observe only two bands tend to measure the R and NIR bands so that the NDVI can be derived. However, the NDVI derives from a period when the availability of sensors was limited and consequently the number and the wavelengths of bands collected were also limited. Nowadays, there are more optical satellite systems available, as well as aerial and proximal canopy sensing systems. Collectively, these provide a much wider range of available wavelengths/bands, which generates many more options for combining bands. Subsequently, there have been a variety of new VIs proposed over the past two decades. For example, normalized difference red edge (NDRE) is a fairly well known VI and is increasingly being used in other crops instead of NDVI (Amaral et al. 2015, Carneiro 2020).

Despite the availability of newer VIs, the NDVI still remains the most common VI used in viticulture studies. A 
Boolean search of articles post-2008 in Scopus returned nearly four times as many hits for "TITLE-ABS-KEY (viticulture AND ndvi)" as for "TITLE-ABS-KEY (viticulture AND ci OR savi OR mtci OR wdrvi OR rvi)" (performed 18 Feb 2021). There have been recent studies comparing the quality and relationship between NDVI imagery obtained from different platforms (terrestrial - unmanned aerial vehicles - satellite) over the same vineyard area (e.g., Borgogno-Mondino et al. 2018, Di Gennaro et al. 2019, Khaliq et al. 2019, Sozzi et al. 2020), under the assumption that the NDVI is the optimum VI for viticulture management. However, very little work has been done on determining if this assumption is correct and if it is universal for all vineyard parameters associated with vine size, yield, and fruit quality. The utility of these alternative VIs in viticulture, in particular for canopy vigor studies, has been poorly explored (Towers et al. 2019). Taskos et al. (2015) reported relationships between different VIs and vine size (pruning weight, $\mathrm{PW}$ ), albeit in only two vineyards over two years with four similar VIs (three versions of NDVI and the chlorophyll index [CI]). Towers et al. (2019) investigated the effect of netting on VI-leaf area index relationships in two vineyards in Argentina for eight VIs. In neither study were clear patterns in VI response and a preferred VI for vine size derived, although the issue of a loss of sensitivity (signal saturation) with the NDVI at larger vine sizes was noted in both. Marciniak et al. (2015) reported that correlations with PW were stronger with green-based VIs than with NDVI. It should be noted that there has been some work also looking at different VI correlations to grape quality parameters (e.g., Ledderhof et al. 2016), but the emphasis here is only on vine size $(\mathrm{PW})$.

Consequently, the question of whether the NDVI is the preferred VI for canopy and vine size management remains largely unanswered in the literature. Given that many of the newer VIs, or combinations of VIs, have been shown to provide effective alternatives to NDVI in other crops (NguyRobertson 2012, Carneiro et al. 2020), their relevance to viticulture needs to be tested, particularly when applied to commercial production situations. In this short study the relationship of seven VIs, including NDVI, to vine size is investigated using a proximal multispectral canopy sensor in multiple commercial Concord (Vitis labruscana cv. Bailey) juice grape vineyards - that is, not in research plots. The objective is to determine if NDVI remains the preferred VI for PW mapping with terrestrial proximal canopy sensing systems.

\section{Materials and Methods}

The data used in this study have been previously described in a protocol for mapping $\mathrm{PW}$ in commercial vineyards in the Lake Erie American Viticultural Area (AVA), New York (Taylor et al. 2017), and readers are directed to this paper for any further clarification. Only blocks with a reasonable trend between NDVI and PW ( $r>0.55)$ were used in this study (15 blocks in 2012 and 12 blocks in 2013).

The Lake Erie AVA is a cool-climate region confined to a narrow strip along the New York and Pennsylvania shore of Lake Erie. Most production is for Concord juice grapes and practices are very uniform in the region with the majority of vines trained to a single high-wire trellis at a row spacing of $\sim 2.7 \mathrm{~m}$ and a vine spacing of $\sim 2.4 \mathrm{~m}$.

Canopy sensing surveys. The protocol of the sensing surveys is described in detail in Taylor et al. (2017). Briefly, surveys were conducted around veraison (when final vine size has typically been reached) using a CropCircle 430 sensor (Holland Scientific) mounted horizontally on a vehicle to sense the canopy side-curtain. Sensing was performed in two- or three-row patterns and georeferenced using a WAASenabled GPS receiver (Garmin 18x, Garmin Ltd.). The CropCircle 430 records three bands centered on 670 (R), 730 (RE), and $780 \mathrm{~nm}$ (NIR) and calculates the NDVI and NDRE indices (see Table 1 for formulas).

As well as NDVI and NDRE, several other VIs were calculated that use combinations of the wavelengths measured by the CropCircle 430 . Names, abbreviations, and formulas for the selected VIs are given in Table 1. Different sensors have different specifications and broadband multispectral VIs tend to be derived from satellite sensors rather than proximal sensors. For all VIs, the central wavelength of the CropCircle 430 bands (R, RE, or NIR) was verified against the original sensor specifications to ensure that it was located within the original wavelength range. The original difference vegetation index (Richardson and Wiegand 1977) had a coefficient to adjust for background soil effects. This coefficient was removed and the VI simplified (Table 1), as the ground is not imaged with this sensor setup. It should be noted that the simplified difference VI (DifVI) is a distance index while all other VIs are ratio indices.

PW data. In each vineyard block, three to five rows were identified that collectively included high, low, and medium NDVI areas in the interpolated NDVI map. Sampling sites were targeted along these rows to collect 20 to $25 \mathrm{PW}$

Table 1 Vegetative indices, their abbreviation, formula, and original citation used in this study.

\begin{tabular}{llll}
\hline Name & Abbreviation & \multicolumn{1}{c}{ Formula } & Reference \\
\hline Normalized difference vegetation index & NDVI & (NIR-R)/(NIR+R) & Rouse et al. 1974 \\
Simplified difference vegetation index & DifVI & NIR - R & Adapted from Richardson and Wiegand 1977 \\
Simple ratio (or Plant cell density) & SR (PCD) & NIR/R & Jordan 1969 \\
Normalized difference red edge & NDRE & (NIR-RE)/(NIR+RE) & Barnes et al. 2000 \\
Modified simple ratio & $\mathrm{MSR}$ & $\mathrm{R} /$ sqrt((NIR/R)+1) & Chen 1996 \\
Red-edge chlorophyll index & $\mathrm{RECl}$ & $(\mathrm{NIR} / \mathrm{RE})-1$ & Gitelson et al. 2003 \\
MERIS terrestrial chlorophyll index & $\mathrm{MTCl}$ & $(\mathrm{NIR}-\mathrm{RE}) /(\mathrm{RE}-\mathrm{R})$ & Dash and Curran 2004 \\
\hline
\end{tabular}


measurements over a range of NDVI values. The PW was a panel measurement between two supporting posts (typically $7.32 \mathrm{~m}$ or three vines), and the panel midpoint was georeferenced as the sample location. Panel measurements were done to simplify the task for the pruning crew and to obtain an area measurement that smooths some of the inherent vine to vine variability (Taylor and Bates 2012). The PW measurements were not constrained to the rows surveyed by the canopy sensor (for further details see Taylor et al. 2017). These constraints were important as the process was implemented in commercial vineyards and often used commercial, not research, staff to collect the data. There was a need to balance the quality of these data and the cost of obtaining it.

Data analysis. The NDVI was considered as the baseline VI for the study. As a first step in analysis, the CropCircle 430 NDVI data for each vineyard were independently assessed for skewness and a saturated response (not observed) before being trimmed $(0.3<\mathrm{NDVI}<1$ and/or values $\pm 3 \sigma$ from the mean). Maps of NDVI within all the vineyards were then generated using a local block kriging approach $(8 \mathrm{~m}$ blocks $=$ typical swathing width) with the shareware Vesper (Minasny et al. 2005). Further information on the interpolation process is given in Taylor et al. (2007). The individual bands (R, RE, and NIR) from the CropCircle 430 output were interpolated onto the PW sample locations using local block kriging $(8 \mathrm{~m}$ blocks). The interpolated band data were then used to generate the VIs at each sampling site using the formulas in Table 1.

The NDVI was plotted against the PW and extreme outliers were removed from the raw data as a first step-that is, sites with a high PW and low NDVI or vice versa, that were likely due to some form of sampling error. Eighteen of 763 collected samples $(2.4 \%)$ were omitted at this stage, which is considered a fairly low exclusion rate for data explicitly collected under commercial production conditions. Note that the trimming was done on the NDVI-PW relationship only.

Following data trimming, a principal components analysis (PCA) was performed on the seven derived VIs on each individual block. The first principal component (PC1) was extracted and stored. PCA is considered a robust and repeatable approach to data reduction (Jolliffe and Cadima 2016). The seven VIs were derived from only three bands and ex- hibit a high level of correlation (typically $r>0.90$, data not shown). One hypothesis is that a multivariate, rather than a univariate, approach is preferable when modeling a crop response with multispectral sensors (Nguy-Robertson et al. 2012). However, modeling multivariate solutions makes model comparison more complex, especially if vineyards have different numbers of VIs to achieve the optimal model. The PCA approach provides an insight into using multiple VIs for PW calibration while maintaining a univariate linear regression model to be compared with the individual VIs.

A final spreadsheet was generated with site ID, vineyard ID, location (Eastings and Northings, UTM WGS84), PW, three bands (interpolated), seven VIs, and PC1.

Comparison of all VIs. Linear regression was performed to fit the PW data to each of the VIs (including PC1) on a vineyard-by-vineyard basis. The adjusted coefficient of regression $\left(\mathrm{R}^{2}{ }_{\text {adj }}\right)$ and root mean square error (RMSE) of the fit in each vineyard were recorded. For each vineyard $(\mathrm{n}=$ 27) the $\mathrm{R}^{2}$ adj of each VI was ranked, such that the VI with the highest $\mathrm{R}^{2}$ adj was given a rank of 1 and the lowest $\mathrm{R}^{2}$ adj $\mathrm{a}$ rank of 8 . The mean rank and the standard deviation of rank were calculated for each VI and plotted (mean rank versus standard deviation of rank). The best-performing VI would have the lowest mean rank and lowest standard deviation of rank. The mean $\mathrm{R}^{2}$ adj for each VI across all vineyards was also calculated. Finally, the "best scenario" $\mathrm{R}^{2}$ adj was calculated by averaging the best fit for each vineyard, regardless of the VI selected.

\section{Results and Discussion}

The number of times each VI was given a particular rank is shown in Table 2. The NDVI did not rank as the best VI in any vineyard (Table 2) and was reasonably well distributed across the other ranks. The NDRE and RECI (RE-based indices) had more scores in the first four rankings than the last four. The PC1 and DifVI were heavily weighted to the midrankings (particularly rank 4 and rank 5, respectively). The PC1 approach was not ranked below 5 in any of the 27 vineyards. The modified simple ratio (MSR), Plant Cell Density or Simple Ratio (PCD/SR), and MERIS terrestrial chlorophyll index (MTCI) were the worst-performing indices. However,

Table 2 Counts of the rank assigned for each vegetative index (VI) ( $n=27$ vineyards) and the mean adjusted coefficient of regression $\left(\mathrm{R}^{2}\right.$ adi) value for each $\mathrm{VI}$ when regressed against vine pruning weight in each individual vineyard. PC1, first principal component; NDRE, normalized difference red edge; DifVI, simplified difference vegetation index; RECI, red-edge chlorophyll index; NDVI, normalized difference vegetation index; MSR, modified simple ratio; MTCI, MERIS terrestrial chlorophyll index; PCD/SR, Plant Cell Density or Simple Ratio.

\begin{tabular}{|c|c|c|c|c|c|c|c|c|c|}
\hline \multirow{2}{*}{$\begin{array}{l}\text { Vegetative } \\
\text { index }\end{array}$} & \multicolumn{8}{|c|}{ Rank } & \multirow[b]{2}{*}{ Mean $\mathbf{R}^{2}$ adj } \\
\hline & 1 & 2 & 3 & 4 & 5 & 6 & 7 & 8 & \\
\hline PC1 & 2 & 2 & 6 & 12 & 5 & 0 & 0 & 0 & 0.332 \\
\hline NDRE & 1 & 7 & 6 & 3 & 2 & 3 & 5 & 0 & 0.313 \\
\hline DifVI & 1 & 3 & 4 & 6 & 10 & 2 & 1 & 0 & 0.326 \\
\hline RECl & 2 & 4 & 4 & 6 & 0 & 8 & 3 & 0 & 0.312 \\
\hline NDVI & 0 & 7 & 4 & 0 & 7 & 3 & 5 & 1 & 0.328 \\
\hline MSR & 6 & 4 & 2 & 0 & 0 & 7 & 2 & 6 & 0.319 \\
\hline $\mathrm{MTCl}$ & 9 & 0 & 0 & 0 & 1 & 0 & 4 & 13 & 0.261 \\
\hline PCD/SR & 6 & 0 & 1 & 0 & 2 & 4 & 7 & 7 & 0.301 \\
\hline
\end{tabular}


these three VIs showed an unusual characteristic in that they either performed very well or very poorly (Table 2). MTCI, for example, had the highest count of both rank 1 and rank 8 , and MSR and PCD/SR had similar trends.

The mean $\mathrm{R}^{2}$ adj for each VI across all vineyards is also shown in Table 2 . The mean $\mathrm{R}_{\text {adj }}$ values were similar across most of the VIs, in the range of 0.312 to 0.332 . The mean $\mathrm{R}^{2}$ adj of the best VI model from each vineyard, regardless of the VI selected, was 0.386. Optimizing the choice of VI in each vineyard provided some small improvement and explained $\sim 6 \%$ more of the variance in PW than the NDVI model. The $\mathrm{R}^{2}{ }_{\text {adj }}$ values were not particularly high in these data and certainly less than those observed in similar studies (Drissi et al. 2009, Stamatiadis et al. 2010). However, this was an expected effect of data collected in commercial situations with a known series of potential errors. Clear spatial patterns in the VIs were still present in all vineyards (data not shown). Taylor et al. (2017) proposed a second level of data trimming to more accurately approximate the strength of the regression fit between NDVI and PW, and the mean $\mathrm{R}_{\text {adj }}^{2}$ for NDVI-PW for these 27 vineyards was 0.480 . This second-level trimming was not performed here because different VIs would likely generate unequal sample sizes that could have a large effect on model statistics, rendering comparisons difficult.

Figure 1 graphically shows the relationship between mean rank and standard deviation of rank for each VI and summarizes the raw data in Table 2. Despite the similarity in mean $\mathrm{R}_{\text {adj }}^{2}$, the $\mathrm{PC1}$ approach had the lowest mean rank and

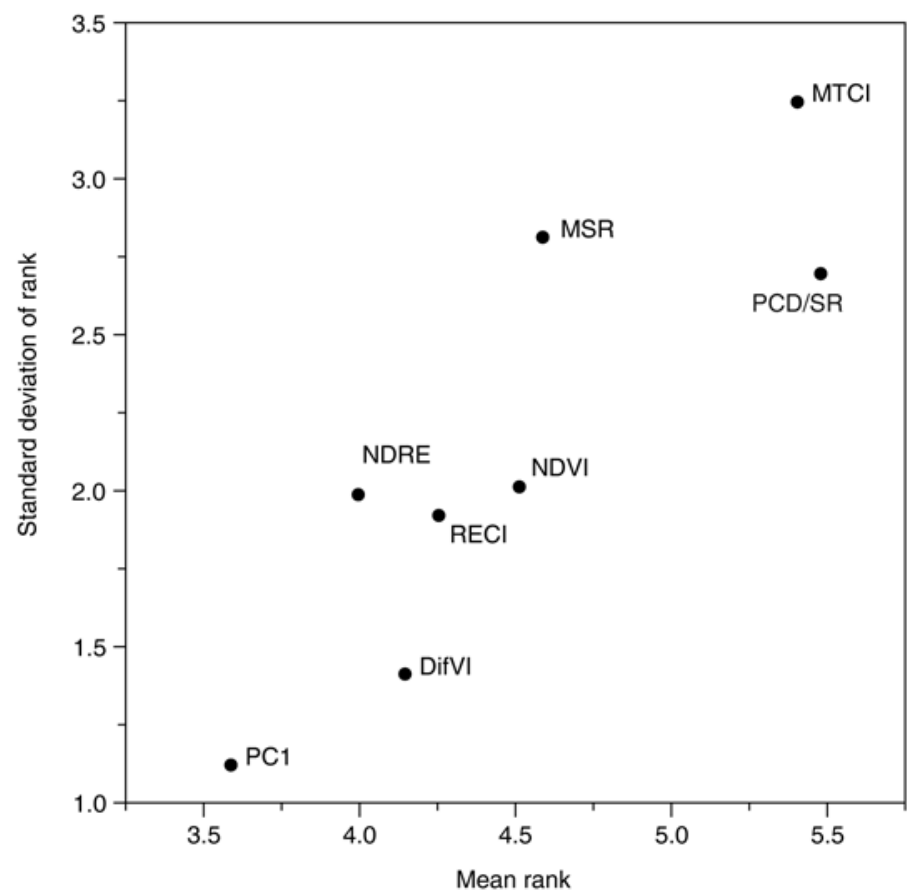

Figure 1 Plot of the mean rank versus the standard deviation of rank for each vegetation index $(\mathrm{VI})$. PC1 refers to the first principal component from a PCA of all seven VIs in Table 1. NDRE, normalized difference red edge; DifVI, simplified difference vegetation index; RECI, red-edge chlorophyll index; NDVI, normalized difference vegetation index; MSR, modified simple ratio; MTCI, MERIS terrestrial chlorophyll index; PCD/ SR, Plant Cell Density or Simple Ratio. lowest standard deviation of rank (Figure 1). In terms of individual VIs, the little-used DifVI performed well. Of the best-performing ratio indices, the RE-based indices of RECI and NDRE performed slightly better (lower mean rank) than the R-based NDVI.

Fitting the different VIs to a common data set across multiple vineyards showed that there was no clear preference to using NDVI, despite the data trimming being based on the NDVI response. Using the PC1 from a PCA of the seven VIs generated the best result, indicating that there is likely to be a benefit from a multi-VI approach. Of the individual VIs, those that used a direct ratio between NIR and RE performed well compared to NDVI. There were two outcomes of particular interest. Firstly, DifVI, which is based on a difference rather than a ratio, had a lower mean rank than all VIs except NDRE, although the DifVI standard deviation was much lower than NDRE. Secondly, the SR (also known as the PCD) had no advantage (Figure 1). The SR has been advocated and used successfully for aerial multispectral canopy sensing by commercial service providers in Australia (Hall et al. 2002) and has been shown to be less affected by signal saturation than NDVI (Ji and Peters 2007). The sensing protocol here, with side-mounted proximal sensors, removed the issue of signal saturation and perhaps the advantage that SR has when imaging the canopy from overhead sensing systems.

It is clear from the results here, especially Table 2, that there is no one single preferred VI. All VIs, with the exception of NDVI, achieved a rank of 1 in at least one vineyard. Fitting all the VIs individually and then using the best fit for modeling appeared to produce an advantage. However, this requires more processing and increases the effort required. From an operational point of view, fully automated modeling is preferable. Switching between VIs makes this more difficult, but such an approach may benefit from emerging machine-learning approaches.

The study here was relatively large for a canopy sensing study (27 individual blocks); however, care should still be used when transferring these results to other production systems, especially other types of trellising. The analysis was limited to PW, and other vineyard traits, including berry quality traits, may have different relationships (Anastasiou et al. 2018). Similarly, canopy data generated from overhead remote systems (satellite or aerial systems) with a nadir (downward) view may not necessarily behave in the same way. Further studies are needed to expand knowledge in this area.

\section{Conclusions}

Many more VIs are now available for use in agriculture. When applied to data from 27 vineyard blocks, there was no single outstanding VI for relating canopy reflectance to PW. However, the results showed that when RE band data are available, the RE:NIR ratio indices had a slight advantage over more common R:NIR ratio indices. However, in terms of model fit and RMSE, the advantage appeared minimal. If a single VI is to be used, then changing to the use of NDRE or RECI is recommended for single high-wire juice grapes, but NDVI still performed well and should be the preferred 
VI in situations when only R and NIR bands are available. In this study, a distance index between the NIR and R response performed well, but this has been poorly investigated to date and requires further testing. Combining bands, in this case by decomposition with PCA, provided the best solution, and more work is required on multi-VI applications to mapping PW. How various VIs respond in other trellis systems, with different (overhead) sensors and to other vineyard traits, still needs further work.

\section{Literature Cited}

Amaral LR, Molin JP, Portz G, Finazzi FB and Cortinove L. 2015. Comparison of crop canopy reflectance sensors used to identify sugarcane biomass and nitrogen status. Precis Agric 16:15-28.

Anastasiou E, Balafoutis A, Darra N, Psiroukis V, Biniari A, Xanthopoulos G and Fountas S. 2018. Satellite and proximal sensing to estimate the yield and quality of table grapes. Agriculture 8:94.

Barnes EM et al. 2000. Coincident detection of crop water stress, nitrogen status and canopy density using ground-based multispectral data. In Proceedings of the Fifth International Conference on Precision Agriculture, Bloomington, MN, USA.

Borgogno-Mondino E, Lessio A, Tarricone L, Novello V and de Palma L. 2018. A comparison between multispectral aerial and satellite imagery in precision viticulture. Precis Agric 19:195-217.

Carneiro FM, Furlani CEA, Zerbato C, Candida de Menezes P, da Silva Gírio LA and de Oliveira MF. 2020. Comparison between vegetation indices for detecting spatial and temporal variabilities in soybean crop using canopy sensors. Precis Agric 21:979-1007.

Chen JM. 1996. Evaluation of vegetation indices and a modified simple ratio for boreal application. Can J Remote Sens 22:229-242.

Dash J and Curran PJ. 2004. The MERIS terrestrial chlorophyll index. Int J Remote Sens 25:5403-5413.

Di Gennaro SF, Dainelli R, Palliotti A, Toscano P, Matese A. 2019. Sentinel-2 validation for spatial variability assessment in overhead trellis system viticulture versus UAV and agronomic data. Remote Sens 11:2573.

Dobrowski SZ, Ustin SL and Wolpert JA. 2003. Grapevine dormant pruning weight prediction using remotely sensed data. Aust J Grape Wine Res 9:177-182.

Drissi R, Goutouly J-P, Forget D, and Gaudillere J-P. 2009. Nondestructive measurement of grapevine leaf area by ground normalized difference vegetation index. Agron J 101:226-231.

Gitelson AA, Viña A, Arkebauer TJ, Rundquist DC, Keydan G and Leavitt B. 2003. Remote estimation of leaf area index and green leaf biomass in maize canopies. Geophys Res Letters 30:1248.

Hall A, Lamb DW, Holzapfel B and Louis J. 2002. Optical remote sensing applications in viticulture- a review. Aust J Grape Wine Res 8:36-47.

Hall A, Louis JP and Lamb DW. 2008. Low-resolution remotely sensed images of winegrape vineyards map spatial variability in planimetric canopy area instead of leaf area index. Aust J Grape Wine Res 14:9-17.

Ji L and Peters AJ. 2007. Performance evaluation of spectral vegetation indices using a statistical sensitivity function. Remote Sens Environ 106:59-65.

Johnson L. 2003. Temporal stability of the NDVI-LAI relationship in a Napa Valley vineyard. Aust J Grape Wine Res 9:96-101.
Jolliffe IT and Cadima J. 2016. Principal component analysis: A review and recent developments. Philos Trans A Math Phys Eng Sci 374:20150202.

Jordan CF. 1969. Derivation of leaf-area index from quality of light on forest floor. Ecology 50:663-666.

Khaliq A, Comba L, Biglia A, Ricauda Aimonino D, Chiaberge M and Gay P. 2019. Comparison of satellite and UAV-based multispectral imagery for vineyard variability assessment. Remote Sens 11:436.

Ledderhof D, Brown R, Reynolds A and Jollineau M. 2016. Using remote sensing to understand Pinot noir vineyard variability in Ontario. Can J Plant Sci 96:89-108.

Marciniak M, Brown R, Reynolds AG and Jollineau M. 2015. Use of GPS, GIS and remote sensing to understand the terroir of the Niagara Peninsula. Remote sensing applications in a Riesling vineyard. J Int. Sci Vigne Vin 49:1-26.

Matese A et al. 2015. Intercomparison of UAV, aircraft and satellite remote sensing platforms for precision viticulture. Remote Sens 7:2971-2990.

Minasny B, McBratney AB and Whelan BM. 2005. VESPER version 1.62. Precision Agriculture Laboratory, Faculty of Agriculture and Environment, The University of Sydney, NSW 2006. (http://sydney. edu.au/agriculture/pal/software/vesper.shtml).

Nguy-Robertson A, Gitelson A, Peng Y, Viña A, Arkebauer T and Rundquist D. 2012. Green leaf area index estimation in maize and soybean: Combining vegetation indices to achieve maximal sensitivity. Agron J 104:1336-1347.

Richardson AJ and Wiegand CL. 1977. Distinguishing vegetation from soil background information. Photogramm Eng Rem S 43:1541-1552.

Rouse JW, Haas RH Jr, Schell JA and Deering DW. 1974. Monitoring vegetation systems in the Great Plains with ERTS. In Proceedings of the Third ERTS-1 Symposium. pp. 309-317. Washington, DC.

Sozzi M, Kayad A, Marinello F, Taylor JA and Tisseyre B. 2020. Comparing vineyard imagery acquired from Sentinel-2 and Unmanned Aerial Vehicle (UAV) platform. OENO One 54:189-197.

Stamatiadis S, Taskos D, Tsadila E, Christofides C, Tsadilas C and Schepers JS. 2010. Comparison of passive and active canopy sensors for the estimation of vine biomass production. Precis Agric 11:306-315.

Taskos DG, Koundouras S, Stamatiadis S, Zioziou E, Nikolaou N, Karakioulakis K and Theodorou N. 2015. Using active canopy sensors and chlorophyll meters to estimate grapevine nitrogen status and productivity. Precis Agric 16:77-98.

Taylor JA and Bates TR. 2012. Sampling and estimating average pruning weights in Concord grapes. Am J Enol Vitic 63:559-563.

Taylor JA, McBratney AB and Whelan BM. 2007. Establishing management classes for broadacre grain production. Agron J 99:1366-1376.

Taylor JA, Nuske S, Singh S, Hoffman JS and Bates TR. 2013. Temporal evolution of within-season vineyard canopy response from a proximal sensing system. In Precision Agriculture '13: Proceedings of the Ninth European Conference on Precision Agriculture. Stafford JV (ed.), pp. 659-665. Lleida, Spain. Wageningen Academic Publishers.

Taylor JA, Link K, Taft T, Jakubowski R, Joy P, Martin M, Hoffman JS, Jankowski J and Bates TR. 2017. A protocol to map vine size in commercial single high-wire trellis vineyards using "off-the-shelf" proximal canopy sensing systems. Catalyst 1:35-47.

Towers PC, Strever A and Poblete-Echeverría C. 2019. Comparison of vegetation indices for leaf area index estimation in vertical shoot positioned vine canopies with and without grenbiule hail-protection netting. Remote Sens 11:1073. 